\title{
Análisis de la utilidad del programa básico de formación docente en modalidad semipresencial
}

\author{
Analysis of the usefulness of the basic program of teacher training \\ in a blended learning modality
}

\author{
Joel Eduardo Vielma-Puente ${ }^{a, b}$, Maria Alejandra Ruano ${ }^{b, c}$ \\ ${ }^{a}$ Escuela Superior Politécnica del Litoral, ESPOL. PhD. En Química Aplicada, \\ Facultad de Ciencias Naturales y Matemáticas, Campus Gustavo Galindo, Ecuador. \\ jvielma@espol.edu.ec \\ ${ }^{b}$ Centro de Investigaciones y Servicios Educativos, CISE. \\ ${ }^{c}$ Escuela Superior Politécnica del Litoral, ESPOL. MSc. Economía, \\ Facultad de Ciencias Sociales y Humanísticas, Campus Gustavo Galindo, Ecuador. \\ maruano@espol.edu.ec
}

\section{RESUMEN}

El objetivo del artículo es evaluar la utilidad del Programa Básico de Formación Docente (PBFD) en la modalidad semipresencial o Blended Learning (BL) en el quehacer docente utilizando un método descriptivo a través de una encuesta transversal con la participación de 35 docentes universitarios. Se utilizó la plataforma edX como aula virtual, en donde los participantes se apoyaron con videos, lecturas, notas técnicas, rúbricas de evaluación y clases presenciales para la formación y medición de los objetivos de aprendizaje del programa. Se aplicó un cuestionario para evaluar la utilidad del programa, la percepción de los participantes de la calidad del aprendizaje obtenido y su utilidad para el desarrollo de sus clases. Los resultados demuestran que la modalidad BL es útil ya que el 77,2\% de los participantes consideran entre muy bueno y excelente su aprendizaje, además el 57,1\% considera que los aprendizajes son pertinentes y aplicables en sus clases.

Palabras claves: Educación alternativa, aprendizaje en línea, tecnologías de la información y de la comunicación, Blended Learning.

\section{ABSTRACT}

The objective of this article is to evaluate the usefulness of the Basic Teacher Training Program in the BlendedLearning (BL) modality for teachers using a descriptive method through a cross-sectional survey with the participation of 35 university teachers. The edX platform was used as a virtual classroom, where the participants had different learning material available such as videos, readings, technical notes, evaluation rubrics, and faceto-face classes for training. The results obtained in the evaluations allowed measuring the program's learning objectives. A questionnaire was applied to evaluate the usefulness of the program, the participants' perception of the quality of the education obtained, and its usefulness for the development of their classes. The results show that the BL modality is beneficial, $77.2 \%$ of the participants consider their learning to be very good and excellent, and $57.1 \%$ consider that the learning is relevant and applicable in their classes.

Key words: Alternative education, blended learning, TICs, online learning, information and communication technologies. 


\section{INTRODUCCIÓN}

La educación universitaria requiere de la formación continua de los responsables de impartir los conocimientos dentro de las aulas de clases; esto para un profesor universitario es un gran reto principalmente por la poca disponibilidad de tiempo debido a su trabajo dentro del proceso de enseñanza-aprendizaje y a otras actividades del quehacer docente propias del entorno universitario como lo son la investigación, las actividades de vínculos con la sociedad, las actividades prácticas como visitas industriales, los trabajos de campo entre otras. Por esta razón cursos en modalidades semipresenciales o a distancia en entornos virtuales han tenido gran acogida en los últimos años en una gran cantidad de universidades a nivel global ya que suponen la posibilidad de desarrollar las actividades en horarios flexibles y que pueden ser ajustados por cada profesor, esto gracias al uso de la internet (Hinojo y Fernández, 2012).

Dentro de las modalidades de trabajo que se destacan utilizando ambientes virtuales podemos mencionar, estudios a distancia de manera on-line, en el que estudiante no asiste de manera presencial a las clases y que hoy en día se encuentra en pleno auge. Esta modalidad de enseñanza presenta una serie de ventajas como lo son: a) aprendizaje autorregulado, b) contacto con Tecnologías de Información y de Comunicación (TICs), c) innovación tecnológica y educativa, d) conciliación laboral y familiar, entre muchas otras más. Sin embargo, la educación a distancia on line, tiene algunas desventajas si la comparamos con la educación presencial, como lo son: a) falta de contacto directo con los compañeros y el profesor, b) riesgo de desorden y mala planificación, c) riesgo del abandono de los estudios si se prolongan por mucho tiempo, por mencionar algunas (García et al., 2007). Por otra parte, para evitar los problemas antes mencionados de la educación on line, han surgido otras modalidades como la semipresencial, mixto o combinada, cuyo término utilizado de manera común es Blended Learning.

Blended Learning (BL) puede ser traducido al español como aprendizaje mixto (Bonk y Graham, 2006), lo que hace referencia al desarrollo de un modelo de aprendizaje que involucra actividades presenciales y no presenciales (Bartolomé, 2008). Sin embargo, otros autores traducen Blended Learning como aprendizaje combinado o incluso lo llaman de otra forma como (hybrid model) modelo híbrido; o aunque la palabra learning se refiere literalmente a aprendizaje, más bien dentro del contexto el término debe hacer referencia al entorno de enseñanza donde ocurre el proceso educativo y por lo tanto otros autores se refieren a este como enseñanza semipresencial, enseñanza mixta, enseñanza combinada o incluso educación flexible (Bartolomé, 2008).

El modelo BL ha sido un éxito debido a la implementación de las Tecnologías de Información y de la Comunicación (TICs) propiciadas por el desarrollo de la informática y del internet, generando ambientes virtuales y una gran variedad de herramientas para el aprendizaje autónomo, cooperativo y la autorregulación de los estudiantes. Este tipo de herramientas ayudan al cumplimiento de los objetivos dentro del proceso de enseñanzaaprendizaje, además de brindar la posibilidad del trabajo a distancia desde cualquier parte del mundo y en cualquier momento (Morán, 2012; Comoglio, 2014; Guzmán et al., 2011; Martin, 2008; Sigalés, 2004). No solo la flexibilidad de los horarios les permite a los participantes organizarse mejor de acuerdo con sus diversas actividades, sino que también pueden escoger el mejor momento para aprender ya que cada individuo aprende mejor en diferentes momentos del día o incluso noche. Esta ventaja genera un impacto positivo en 
los resultados de aprendizaje (Stein y Graham, 2020). Además, muchos autores (García y Carbero, 2016; Sadeck, 2016; Stoltenkamp, 2012) exponen que la educación en ambientes virtuales que involucra e-learning se vislumbran como modalidades de aprendizaje que atraen a los estudiantes de todos los niveles.

Para la formación continua de los profesores universitarios y propiciar la innovación del docente en Guayaquil-Ecuador, el Centro de Investigaciones y Servicios Educativos (CISE) creó el Programa Básico de Formación Docente (PBFD) en la Escuela Superior Politécnica del Litoral (ESPOL), similar a lo reportado previamente (Salinas, 2004). Este programa tiene dos versiones, una presencial y a partir del 2017 se implementó la modalidad Blended Learning. Esta última se desarrolla en la plataforma de gestión de contenidos edX (Learning Management System) y además también consta con sesiones presenciales dictadas en las instalaciones universitarias.

Actualmente el PBFD está estructurado de la siguiente manera:

- Módulo cero (0), no evaluado, acredita seis (6) horas académicas y permite que el docente se familiarice con la plataforma edX, así como con otras herramientas digitales que se usan a lo largo de los módulos.

- Siete (7) módulos donde se imparten los conocimientos para la mejora del docente acreditando 24 horas por cada módulo.

- Dos (2) procesos de aplicación práctica. El primero acredita ocho (8) horas mientras que el segundo acredita diez (10) horas.

En la modalidad semipresencial estos módulos son desarrollados en tres semanas, las actividades se habilitan los viernes de cada semana. Por otro lado, cada módulo tiene una clase presencial de cuatro horas, excepto el módulo cuatro que es completamente en línea, sin embargo, tiene designado un tutor al igual que el resto de los módulos.

Los dos procesos de aplicación involucran:

- Revisión curricular en las Unidades Académicas: este proceso acredita 8 horas académicas y consiste en realizar una revisión del Syllabus (Contenido del curso) de su materia. Este trabajo es colaborativo pues el docente junto con su jefe académico superior evalúa la pertinencia y coherencia del contenido para las mejoras del mismo. De esta forma el docente puede retroalimentar dicho contenido para posteriormente realizar las correcciones en el sistema. Para evidenciar este trabajo, el docente elabora un informe donde detalla las sugerencias para la mejora del contenido en base a su revisión.

- Observación entre pares académicos: este proceso acredita 10 horas, aquí el docente es evaluado por un par académico del área (quien también es participante del programa) mediante una clase presencial. Esta clase presencial es realizada durante la jornada laboral del docente, por lo que los estudiantes también están presentes. El docente observado que dicta la clase debe aplicar los aprendizajes logrados durante el programa, mientras que el par académico debe brindar una retroalimentación en base a una rúbrica de desempeño a su compañero, recopilar evidencias (fotos, vídeos) y realizar un informe con el fin de dar a conocer las posibilidades de mejora. En ciertos casos asiste un experto del CISE y el equipo de producción audiovisual para dar mayor retroalimentación a la clase y evidenciar el 
trabajo de la observación, respectivamente. Esta observación se la realiza al finalizar todos los módulos.

Las políticas de aprobación establecidas indican que, para aprobar cada módulo, el participante debe obtener como mínimo 70 puntos sobre 100 .

Las actividades que se realizan en la plataforma están diseñadas para que, a través de lecturas, videos, notas técnicas, trabajo colaborativo, y otras herramientas (Screencasto-matic, Plickers, Playposit, Padlet, entre otras) los docentes mejoren sus habilidades y comprendan su papel dentro del proceso enseñanza-aprendizaje (Del Moral y Villalustre, 2012). Cada módulo dura tres semanas y es diseñado por un experto en el área del conocimiento que se imparte. Por otra parte, los docentes participantes en el curso son apoyados por un tutor quien interactúa con ellos en un proceso de acompañamiento estimulando el cumplimiento de los objetivos a lo largo de todo el programa, a través de foros, chats o correos dentro de la misma plataforma edX. Todas las actividades son calificadas y retroalimentadas a través de la plataforma. Adicional, los participantes deben asistir a una sesión presencial por módulo. Dicha sesión dura 4 horas y se la dicta con el fin de reforzar los conocimientos y atender las consultas de los participantes, así mismo permite expandir la explicación de ciertas actividades que los participantes realizarán en la plataforma a lo largo de la semana. Este seguimiento genera el espacio oportuno y directo para la discusión de las actividades y la evaluación de estas para la consolidación de los conocimientos (Segura-Robles y Gallardo-Vigil, 2013 y Stoltenkamp et al., 2017).

Figura 1. Estructura del Programa Básico de Formación Docente

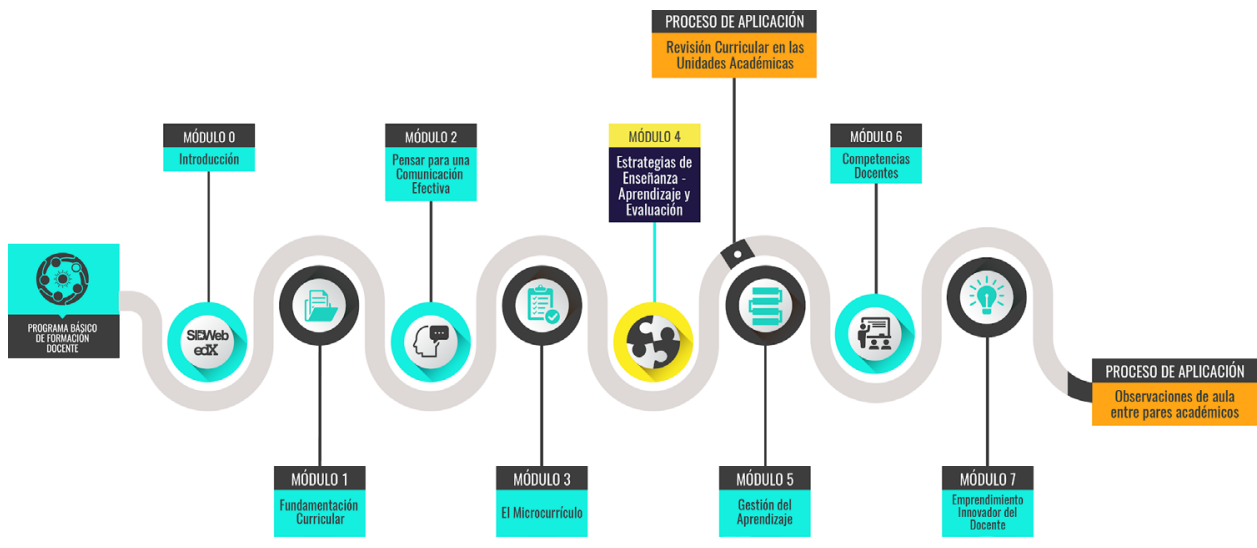

Fuente. (Centro de Investigaciones y Servicios Educativos, 2019).

En el presente trabajo se realizó un estudio de la percepción que tienen los participantes que culminaron el programa con respecto a su utilidad en el aprendizaje de nuevos conocimientos en el área docente y la implementación de estos en el aula de clases.

Los resultados demuestran que el Programa Básico de Formación Docente en la modalidad Blended Learning es una alternativa viable para el desarrollo del proceso de 
enseñanza-aprendizaje ya que presenta resultados favorables y además los participantes consideran que los conocimientos adquiridos son útiles y se pueden implementar en sus aulas de clases. Los resultados hallados son similares a los reportados previamente (SuárezDíaz, 2016; Vera et al., 2014).

El resto del documento se organiza de la siguiente manera: en la sección 2 se presenta el método, en la sección 3 se analizan los resultados y la discusión de estos. Finalmente, en la sección 4 se presentan las conclusiones.

\section{MÉTODO}

\subsection{DISEÑO}

Se realizó un estudio transversal descriptivo con aplicación de una encuesta en una sola ocasión. La encuesta tuvo dos secciones. La primera sección evaluó la percepción de la utilidad del aprendizaje y en la segunda sección se preguntaron los datos demográficos. Esta encuesta fue ejecutada después de la participación de los profesores de la ESPOL en el PBFD, que tiene una duración aproximada de 8 meses. Los participantes tardaron en responder el cuestionario ocho (8) minutos en promedio aproximadamente. La muestra fue recogida durante dos semanas desde el 11 de marzo hasta el 21 de marzo del 2019. En total 35 encuestas fueron efectuadas.

\subsection{POBLACIÓN}

La población de estudio fueron docentes de la ESPOL que culminaron todos los módulos del PBFD (35 participantes) durante el periodo 2018-2019.

\subsection{INTERVENCIÓN}

Los participantes del PBFD intervinieron en este programa en la modalidad semipresencial, en la que se realizó una clase presencial intermedia de cuatro horas en cada módulo exceptuando el módulo 4, en estas se realizaron exposiciones de los temas de interés por parte del tutor académico y actividades grupales de análisis de casos y exposición de dichos análisis. Estas actividades fueron evaluadas mediante rúbricas que se socializaron previamente con los participantes con la finalidad de reforzar temas complejos y dar espacio a una interacción presencial. En esta modalidad semipresencial la mayor parte del trabajo (aproximadamente 90\%) se realizó en un aula virtual, dentro de la plataforma edX, en la que los estudiantes participaron en actividades individuales y grupales que fueron autoevaluadas, evaluadas por el tutor académico y/o por otro estudiante co-evaluador utilizando las rúbricas diseñadas para cada actividad y que fueron socializadas a todos los participantes previamente a la entrega de las actividades.

\subsection{INSTRUMENTO}

Se aplicó un cuestionario diseñado por los autores del presente trabajo en forma sincrónica y nominativa a los participantes del PBFD en la modalidad BL. 


\subsection{ASPECTOS ÉTICOS}

El cuestionario de preguntas fue enviado posterior a la culminación del PBFD a todos los participantes, y se indicó que se respetaría la confidencialidad de las respuestas y no se requirió la identificación del participante.

\section{RESULTADOS Y DISCUSIÓN}

La encuesta fue respondida por 35 participantes de los cuales el 55,9\% eran hombres y el $44,1 \%$ mujeres.

En la encuesta se evaluaron los siguientes aspectos:

- ¿Cómo calificaría usted su aprendizaje en el PBFD?

- ¿Considera que los aprendizajes adquiridos en el PBFD son aplicables en su clase?

- ¿Cuáles de los temas que cursó en el PBFD ayudaron a fortalecer su práctica docente y cuales ha utilizado en sus clases?

- ¿Qué aspectos de lo aprendido en el PBFD considera ha impactado de manera positiva en el aprendizaje de sus estudiantes?

- ¿Qué técnicas de enseñanza utilizada en el PBFD considera útil en su quehacer docente?

- ¿Qué factores considera necesarios para culminar de manera satisfactoria el PBFD en la modalidad Blended Learning?

A continuación, se presentan los resultados obtenidos de cada uno de los aspectos evaluados en la encuesta.

El 40\% de los participantes considera que su aprendizaje en el PBFD es muy bueno, el $35 \%$ que es excelente y el $25 \%$ que es bueno, ningún participante calificó el aprendizaje como malo o muy malo (Figura 2).

Figura 2. Calificación del aprendizaje por parte de los participantes en el PBFD.

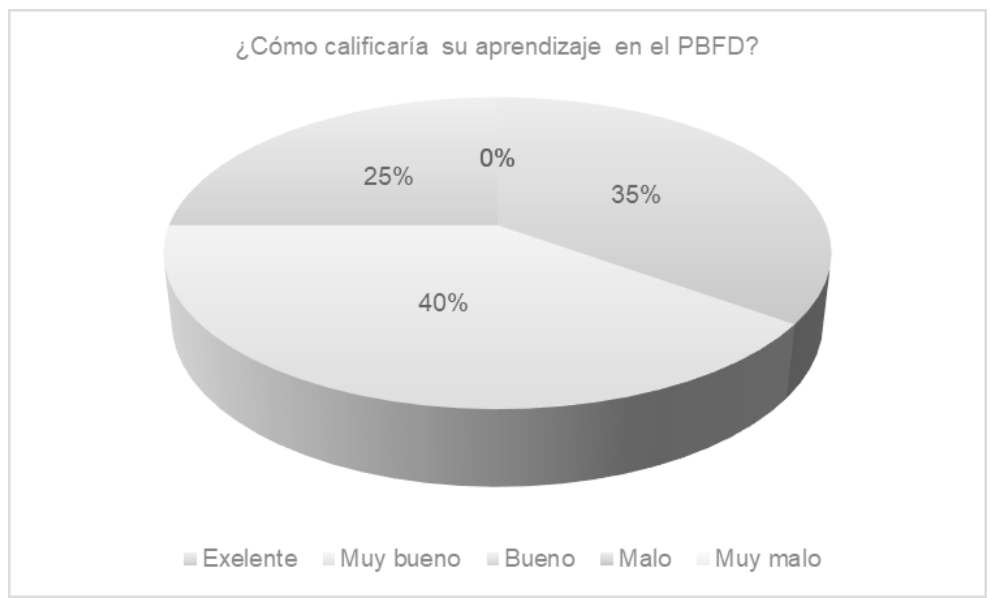


Uno de los aspectos más relevantes de esta investigación fue encontrar que los conocimientos desarrollados en el PBFD son aplicables en diferentes ambientes de aprendizaje en la Educación Superior en modalidad presencial, lo cual es un reflejo de la utilidad de este programa. Los resultados que se aprecian en la Figura 3 muestran que el $90 \%$ de los participantes opinan que los aprendizajes adquiridos son aplicables en sus aulas de clases.

Figura 3. Aplicabilidad de los aprendizajes adquiridos en el PBFD.

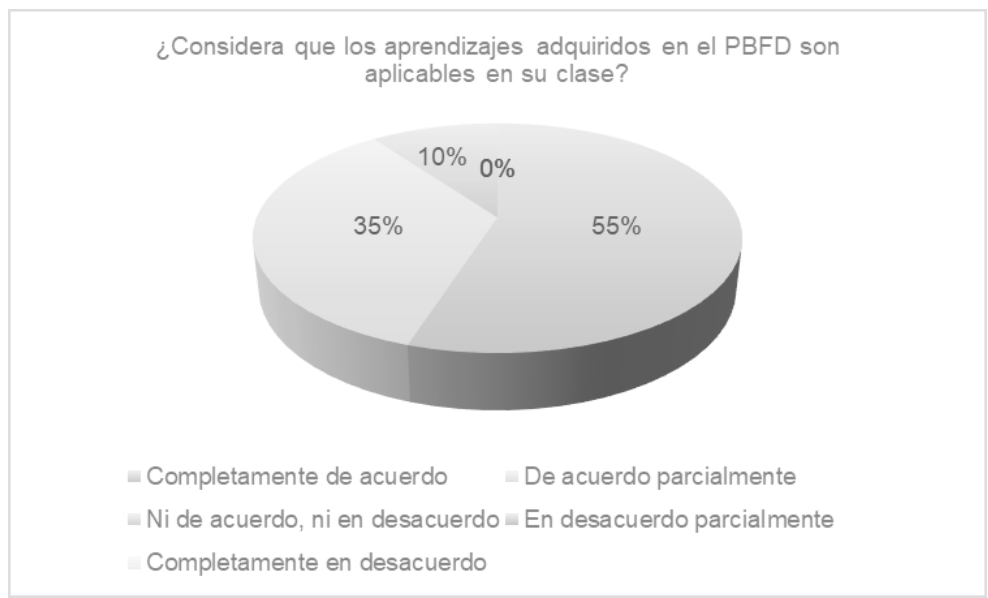

El PBFD aborda los siguientes temas dentro del área de la educación universitaria:

1. Pertinencia en la Educación Superior

2. Ética institucional

3. Estrategias de comprensión lectora

4. Formulación de Objetivos

5. Planificación microcurricular: el sílabo y el programa analítico

6. Técnicas didácticas

7. Herramientas tecnológicas

8. Habilidades docentes

9. Instrumentos de evaluación

10. Emprendimiento innovador del docente

En el análisis de los temas mencionados que han contribuido en mayor medida la práctica docente de los participantes determinó que: Habilidades Docentes, Herramientas Tecnológicas y Formulación de Objetivos son los temas que consideran han fortalecido en mayor medida su práctica docente, evidenciando un nivel de aprobación del $80 \%$. También es importante resaltar que los temas que consideran que menos han fortalecido la práctica docente son Pertinencia de la Educación Superior y Emprendimiento Innovador del Docente con un nivel de aprobación mayor al 50\%, lo que indica que en general todos los 
temas abordados contribuyen en alguna medida en la práctica docente y además demuestra la pertinencia de los conocimientos impartidos durante el programa.

De la variedad de recursos trabajados durante el PBFD, los participantes mencionan que los instrumentos de evaluación (rúbricas, lista de chequeos u otros) y las herramientas digitales (Kahoot, Plickers, Padlet u otros) son los más utilizados en sus clases luego de haber culminado el programa, por lo que se infiere el desarrollo de una evaluación de los aprendizajes más objetiva y precisa, y que como es de esperar en esta nueva era, las TICs juegan un papel importante para gestionar los conocimientos dentro y fuera de las aulas de clases.

Una vez concluido el PBFD y aplicado los conocimientos logrados en su quehacer docente, los participantes consideran que el aspecto que ha impactado de manera positiva y en mayor medida en el aprendizaje de los estudiantes es la comunicación asertiva, seguido de las herramientas de evaluación. La comunicación asertiva con cada estudiante utilizando la empatía, concuerda con el modelo educativo de la institución, que está centrado en el estudiante y tiene como objetivo conseguir la excelencia académica.

Respecto a la utilidad de las técnicas de enseñanza los participantes del PBFD resaltan el uso de videos informativos, seguido del uso de mapas conceptuales o mentales.

Por otra parte, se pudo apreciar que los participantes del PBFD en modalidad BL, están satisfechos con lo aprendido e indican que de manera cualitativa han apreciado mejoras en los resultados en sus estudiantes, al compararlos con los resultados obtenidos en semestres anteriores a la realización del PBFD. Dentro de las mejoras apreciadas indican que los estudiantes se muestran más participativos y motivados en sus clases, lo que genera un ambiente propicio para la práctica docente, además indican que ellos se sienten más seguros de la forma en que dictan sus clases y que los mismos estudiantes les han manifestado que sus clases son de calidad y que los conocimientos que se imparten son aprendidos de manera fácil y didáctica. Los participantes también mencionan que han utilizado a lo largo del semestre diferentes herramientas tecnológicas como aplicaciones para celulares, así como actividades grupales tradicionales como debates o talleres para fomentar la participación de sus estudiantes durante la clase y concluyen que los estudiantes muestran gran acogida a este tipo de iniciativas.

Finalmente consideran que los requisitos más importantes para culminar con éxito este programa en la modalidad semipresencial son la automotivación y la dedicación.

\section{CONCLUSIONES}

La formación continua es fundamental en la vida de todo ser humano. Más aún cuando la labor de dicho individuo es ser el canal para que el conocimiento se multiplique. No basta con enseñar, también se debe evaluar que el aprendizaje sea útil. Es por esto que en el presente trabajo se realizó un estudio de la percepción que tienen los docentes de la Escuela Superior Politécnica del Litoral que culminaron el PBFD en la modalidad Blended Learning con respecto a su utilidad en el aprendizaje de nuevos conocimientos en el área docente y su implementación en el aula de clases. Este programa, de modalidad semipresencial, consta de ocho (8) módulos y dos (2) procesos de aplicación práctica. Cada módulo tiene una duración de tres (3) semanas donde los docentes realizan sus actividades autónomas en la plataforma edX. Adicionalmente, durante cada módulo hay una sesión presencial 
obligatoria de cuatro (4) horas con el fin de reforzar los conocimientos y resolver dudas de los participantes. Un tutor los acompaña a lo largo del programa, quien califica las tareas en la plataforma y brinda la retroalimentación necesaria para alcanzar los objetivos planteados en cada módulo. El tutor también guía la sesión presencial, quien junto al diseñador del curso preparan el material a dictar durante esa jornada. La sesión presencial busca reforzar los conocimientos adquiridos y generar un espacio donde los docentes puedan solventar sus dudas y compartir experiencias.

En base a esta investigación se encontró que el PBFD en modalidad educativa semipresencial, tuvo una buena aceptación por parte de los docentes de la institución de educación superior, quienes consideran que el programa es útil y brinda herramientas importantes para mejorar su quehacer docente, además consideran que los temas tratados son pertinentes. Particularmente, los participantes indicaron que los instrumentos de evaluación (rúbricas, lista de chequeos u otros) y las herramientas digitales (Kahoot, Plickers, Padlet u otros) son los más utilizados en sus clases luego de haber culminado el programa.

Nuestros resultados son útiles para los diseñadores de políticas públicas de la institución, ya que, conocer la utilidad del programa, sirve como referencia para la continuidad y mejora del mismo, además permite su expansión a otras instituciones de educación que deseen fortalecer los conocimientos y aptitudes de su personal docente.

Futuros estudios podrían comparar la eficacia del PBFD en su versión semipresencial versus la presencial con el fin de evidenciar las ventajas y desventajas de la educación en línea. Otros estudios se podrían centrar en la utilidad del acompañamiento de la tutoría y la importancia de la retroalimentación oportuna.

\section{REFERENCIAS BIBLIOGRÁFICAS}

Bartolomé, A. (2008). Entornos de aprendizaje mixto en educación superior. Revista Iberoamericana de Educación a Distancia, 11(1), 15-51. doi 10.5944/ried.1.11.955

Bonk, C. \& Graham, C. (2006). The handbook of the blended learning: Global perspectives, local designs. New Jersey: John Wiley and Sons, Inc.

Centro de Investigaciones y Servicios Educativos (CISE). (2020). Programa básico de formación docente. Última visita 23 de junio de 2020. Recuperado desde http://www.cise.espol.edu.ec/es/ programa-b\%C3\%A1 sico-de-formaci\%C3\%B3n-docente-pbfd

Comoglio, M. (2014). Impacto de la integración de tecnologías de la información y la comunicación (TIC) en la enseñanza universitaria en la modalidad blended learning. El caso de la Facultad de Ingeniería de la UNLZ. [tesis Maestría, Universidad Nacional de Lanús]. Última visita 23 de junio de 2020. Recuperado desde http://www.repositoriojmr.unla.edu.ar/descarga/Tesis/ MAMIC/Comoglio_M_Impacto_2014.pdf

Del Moral, M. y Villalustre, L. (2012). Didáctica universitaria en la era 2.0: competencias docentes en campus virtuales. Revista de Universidad y Sociedad del Conocimiento, 9(1), 36-50. Recuperado desde https://www.redalyc.org/pdf/780/78023415004.pdf

García, L., Ruiz, M. y Domínguez, D. (2007). De la educación a distancia a la educación virtual. España: Editorial Ariel SA.

García, C. y Cabero, J. (2016). Evolución y estado actual del e-learning en la formación profesional española. Revista Iberoamericana de Educación a Distancia, 19(2), 167-191. Recuperado desde http://dx.doi.org/10.5944/ried.19.2.15800 
Guzmán, T., García, M. T., Chaparro, R. y Espuny, C. (2011). Formación docente para la integración de las TIC en la práctica educativa. Apertura. Revista de Innovación Educativa, 3(1), 6-13. Recuperado desde http://www.udgvirtual.udg.mx/apertura/index.php/apertura/article/view/181

Hinojo, M. y Fernández, A. (2012). El aprendizaje semipresencial o virtual: nueva metodología de aprendizaje en educación superior. Revista Latinoamericana de Ciencias Sociales, Niñez y Juventud, 10(1), 159-167. Recuperado desde http://www.scielo.org.co/pdf/rlcs/v10n1/ v10n1a09.pdf

Martín, A. (2008). Las TIC en la formación del maestro. "realfabetización" digital del profesorado. Revista interuniversitaria de formación del profesorado, 63(3), 191-206. Recuperado desde https://www.redalyc.org/pdf/274/27418813011.pdf

Morán, L. (2012). Blended learning. Desafío y oportunidad para la educación actual. Revista Electrónica de Tecnología Educativa, 39(1), 1-19. Doi 10.21559/edutec.2012.39.371

Paredes, J. (2009). Tecnología educativa: La formación del profesorado en la era de Internet. España: Aljibe.

Sadeck, O. (2016). An exploration of e-learning practices of teachers at selected schools in the Western Cape [tesis Doctoral, Cape Peninsula University of Technology]. Última visita 23 de junio de 2020. Recuperado desde http://etd.cput.ac.za/bitstream/handle/20.500.11838/2419/209239395Sadeck-OG-Dtech-Informatics-FID-2016.pdf?isAllowed=y\&sequence $=1$

Salinas, J. (2004). Innovación docente y uso de las TIC en la enseñanza universitaria. Revista de Universidad y Sociedad del Conocimiento, 1(1), 1-16. Recuperado desde https://www.redalyc. org/pdf/780/78011256001.pdf

Segura-Robles, A. y Gallardo-Vigil, M. (2013). Entornos virtuales de aprendizaje: Nuevos retos educativos. Revista científica electrónica de Educación y Comunicación en la Sociedad del Conocimiento, 13(2), 260-272. Recuperado desde http://www.eticanet.org/revista/index.php/ eticanet/article/view/34/30

Sigalés, C. (2004). Formación universitaria y TIC: nuevos usos y nuevos roles. Revista de Universidad y Sociedad del Conocimiento, 1(1), 1-6. Recuperado desde https://www.redalyc. org/pdf/780/78011256004.pdf

Stein, J. \& Graham, C. (2020). Essentials for blended learning: A standards-based guide. Routledge.

Stoltenkamp, J. (2012). An integrated approach to e-learning implementation in a complex higher education setting: A case study of the University of the Western Cape [Tesis Doctoral, University of Western Cape]. Última visita 23 de junio de 2020. Recuperado desde https://etd.uwc.ac.za/ xmlui/handle/11394/3410

Stoltenkamp, J., van de Heyde, V. \& Siebrits, A. (2017). ePedagogy as a threshold concept: a reflection on a programme for Science Teaching Assistants. International Journal for Academic Development, 25(2), 195-199. doi 10.1080/1360144X.2017.1375412

Suárez-Díaz, G. (2016). Co-enseñanza: concepciones y prácticas en profesores de una Facultad de Educación en Perú. Revista electrónica de investigación educativa, 18(1), 166-182. Recuperado desde http://redie.uabc.mx/redie/article/view/786

Vera, J., Torres, L. y Martínez, E. (2014). Evaluación de competencias básicas en TIC en docentes de educación superior en México. Píxel-Bit. Revista de Medios y Educación, 44(1), 143-155. Recuperado desde https://www.redalyc.org/pdf/368/36829340010.pdf 